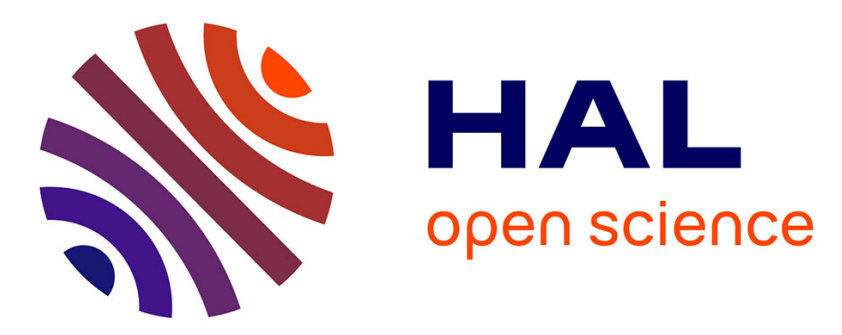

\title{
Aspects thermodynamiques du comportement en fluage biaxial de l'aluminium (99,5\% en poids) à haute température
}

\author{
Ch. Lexcellent
}

\section{- To cite this version:}

Ch. Lexcellent. Aspects thermodynamiques du comportement en fluage biaxial de l'aluminium (99,5\% en poids) à haute température. Revue de Physique Appliquée, 1984, 19 (6), pp.425-430. 10.1051/rphysap:01984001906042500 . jpa-00245212

\section{HAL Id: jpa-00245212 https://hal.science/jpa-00245212}

Submitted on 1 Jan 1984

HAL is a multi-disciplinary open access archive for the deposit and dissemination of scientific research documents, whether they are published or not. The documents may come from teaching and research institutions in France or abroad, or from public or private research centers.
L'archive ouverte pluridisciplinaire HAL, est destinée au dépôt et à la diffusion de documents scientifiques de niveau recherche, publiés ou non, émanant des établissements d'enseignement et de recherche français ou étrangers, des laboratoires publics ou privés. 


\title{
REVUE DE PHYSIQUE APPLIQUÉE
}

Revue Phys. Appl. 19 (1984) 425-430

JUIN 1984, PAGE 425

Classification

Physics Abstracts

$80.00-81.40 \mathrm{~L}$

\section{Aspects thermodynamiques du comportement en fluage biaxial de l'aluminium $(99,5 \%$ en poids) à haute température}

\author{
Ch. Lexcellent \\ Laboratoire de Mécanique Appliquée associé au CNRS, \\ Faculté des Sciences et des Techniques, Route de Gray, La Bouloie, 25030 Besançon Cedex, France
}

(Reçu le 14 novembre 1983, accepté le 14 mars 1984)

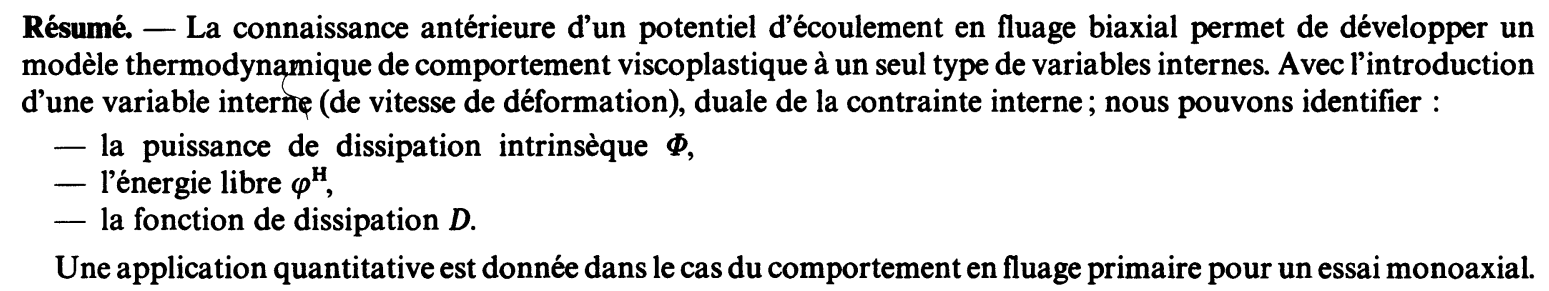

Abstract. - The anterior knowledge of a flow potential in biaxial creep tests, allows us to develop a thermodynamic model for viscoplastic behaviour with only one type of internal variables. With the introduction of an internal variable of deformation rate dual of internal stress, we can identify :

- the intrinsic dissipative power $\Phi$,

- the free energy $\varphi^{\mathbf{H}}$,

- the dissipative function $D$.

We make a quantitative application for primary creep behaviour's for a monoaxial test.

\section{Introduction.}

Dans une récente publication [1], nous avons identifié le comportement viscoplastique en fluage biaxial (traction $\sigma_{z z}$ et torsion $\left.\sigma_{z \theta}\right)$ de l'aluminium $(99,5 \%$ en poids) à haute température $\left(350^{\circ} \mathrm{C}\right)$.

Ce comportement a été interprété en terme d'écrouissage cinématique avec seulement un type de variable interne. Ces variables internes sont reliées au niveau microscopique par la densité moyenne de dislocations (modèle fluage restauration de Lagneborg [2]). Avec Mandel [3], nous supposerons que l'ensemble de toutes les valeurs au temps $t$ des contraintes internes et des variables commandées (les contraintes externes appliquées) donnent complètement l'état de l'échantillon à $t$, incluant les effets de l'histoire antérieure de déformation. Cette hypothèse a été démontrée en fluage monoaxial [4]. Elle exclut la prise en compte de la plasticité instantanée.

Le but de ce travail est d'utiliser les acquis de la thermodynamique avec variables internes faites par Coleman et Gurtin [5] pour les appliquer au cas du fluage biaxial de l'aluminium, nous identifierons ainsi les fonctions thermodynamiques :

- la puissance intrinsèque de dissipation $\Phi$,

- l'énergie libre $\varphi^{\mathbf{H}}$,

- la fonction de dissipation $D$.

Nous chercherons à définir les variables internes de déformation duale aux contraintes internes et à comprendre leur signification physique.

Pour l'aluminium A5, cette approche sera faite pour des chargements proportionnels en fluage biaxial et nous donnerons une application quantitative pour le fluage primaire en sollicitation monoaxiale. 
1. Modèle thermodynamique du comportement viscoplastique à un seul type de variable interne.

1.1 Puissance InTRInsèQue DissipéE. - Soit un élément de matière soumis à un processus de déformation viscoplastique infinitésimal permettant de passer de 1 à 1', l'élément est en équilibre sous l'effet conjugué des contraintes externes et des contraintes internes.

La différence entre un processus réel et un processus réversible purement conceptuel (les forces internes associées aux paramètres internes sont équilibrées par des forces externes fictives) donne le travail mécanique dissipé sous forme de production d'entropie $s^{(i)}[6]$ :

$$
\rho T \mathrm{~d} s^{(i)}=\mathrm{d} W^{D}=\sigma_{i j} \mathrm{~d} \varepsilon_{i j}-X_{i j} \mathrm{~d} a_{i j} \geqq 0
$$

alors la puissance intrinsèque dissipée s'écrit :

$$
\Phi=\dot{W}^{(D)}=\sigma_{i j} \dot{\varepsilon}_{i j}-X_{i j} \dot{a}_{i j} \geqq 0
$$

où $\dot{a}_{i j}$ non encore explicitée est la variable interne de déformation duale de la contrainte interne $X_{i j}$.

1.2 DÉTERMINATION DE L'ÉNERGIE LIBRE D'HELMOLTZ. $-\left\{T, \varepsilon_{i j}^{\mathrm{el}}, a_{i j}\right\}$ est l'ensemble des variables indépendantes pour la fonction d'énergie libre.

Pour un essai isotherme, avec non prise en compte de la déformation e astıque, $\varphi$ evient seu ement fonction des $a_{i j}$ :

$$
\varphi=\varphi\left(a_{i j}\right)
$$

avec :

$$
X_{i j}=\rho \frac{\partial \varphi^{\mathbf{H}}}{\partial a_{i j}}
$$

ce qui donne dans ce cas :

$$
\rho \mathrm{d} \varphi^{\mathbf{H}}=\rho \frac{\partial \varphi^{\mathbf{H}}}{\partial a_{i j}} \mathrm{~d} a_{i j}=X_{i j} \mathrm{~d} a_{i j}
$$

Le bilan énergétique se traduit par le fait que la composante viscoplastique de l'énergie libre est égale à la différence entre le travail viscoplastique effectué et le travail dissipé :

$$
\rho \varphi^{\mathrm{H}}=\int_{0}^{t} \sigma_{i j} \mathrm{~d} \varepsilon_{i j}-\int_{0}^{t} \Phi \mathrm{d} t
$$

\section{Application au fluage biaxial (haute température) de l'aluminium.}

En partant de l'expression de la puissance intrinsèque dissipée, nous pouvons préciser davantage la forme des relations de comportement et d'évolution, si nous faisons l'hypothèse de la dissipativité normale.

Cette hypothèse se traduit par l'usuel « postulat de normalité » non seulement en regard de la vitesse des paramètres externes $\dot{\varepsilon}^{\mathrm{vp}}$ mais aussi en regard des paramètres internes et des forces irréversibles associées.

C'est dans cette logique qu'un potentiel d'écoulement stationnaire $\Omega\left(\sigma_{i j}, X_{i j}, T\right)$ a été identifié [1].

A la suite des travaux de Ponter et Leckie [7] et de Rice [8], nous avons proposé une forme de potentiel

Orowan [9] (modèle fluage-restauration) comme étant applicable à une région de bande de glissement donnée d'une structure homogène de dislocations.

Les formes spécifiques des fonctions-potentiels ont été testées pour des alliages de réacteurs nucléaires [10] (particulièrement le $21 / 4 \mathrm{Cr}-1 \mathrm{Mo}-\mathrm{Fe}$ ).

Une telle forme pour l'aluminium [1] est la suivante :

$$
\Omega\left(\sigma_{i j}, X_{i j}\right)=\frac{K}{n+1}\left[\frac{3}{2}\left(\sigma_{i j}^{\prime}-X_{i j}^{\prime}\right)\left(\sigma_{i j}^{\prime}-X_{i j}^{\prime}\right)-Y^{2}\right]^{\frac{n+1}{2}}+\frac{C}{k+1}\left[\frac{3}{2}\left(X_{i j}^{\prime} X_{i j}^{\prime}\right)\right]^{\frac{k+1}{2}}
$$

avec $n=2$ et $k=5$,

où $K, C$ paramètres du matériau, $Y$ limite élastique proche de zéro pour l'aluminium A5 haute température :

$$
\Omega=\frac{K}{n+1}[\bar{q}]^{n+1}+\frac{C}{k+1}[\bar{X}]^{k+1}
$$

La règle d'écoulement associée à l'équation (7) est :

$$
\dot{\varepsilon}_{i j}=\frac{\partial \Omega}{\partial \sigma_{i j}}=\frac{3}{2} K[\bar{q}]^{n} \frac{q_{i j}^{\prime}}{q}
$$

première règle de normalité.
En suivant la référence [7], la deuxième règle de normalité est la traduction de la loi de croissance de la contrainte interne et donnée par :

$$
\dot{X}_{i j}^{\prime}=-h \frac{\partial \Omega}{\partial X_{i j}}
$$

avec $h$ paramètre d'écrouissage, où l'analyse thẹrmodynamique de Mandel permet d'identifier $\dot{a}_{i j}$ à $\dot{X}_{i j}^{\prime} / h$ :

$$
\frac{\dot{X}_{i j}}{h}=\dot{a_{i j}}=\frac{\partial \Omega}{\partial\left(-X_{i j}\right)}
$$


Le potentiel d'écoulement [1] a été déterminé en fluage stationnaire. Ce fait limite la variance du phénomène car lorsque les contraintes externes sont fixées, dans l'état stationnaire pour une histoire antérieure fixée, les contraintes internes, les vitesses d'écoulement sont fixées... Cela entraîne une difficulté à séparer les variables mises en jeu dans la déformation viscoplastique.

Ainsi, vu que les mesures fiables de $h$ et de $r$ (coefficient de restauration), ne sont possibles qu'à l'état stationnaire, il est difficile de savoir de quelles variables dépendent ces paramètres :

$$
\begin{gathered}
X_{i j}=\rho \frac{\partial \varphi^{\mathrm{H}}}{\partial a_{i j}} \\
\Rightarrow \dot{X}_{i j}=\rho \frac{\mathrm{d}}{\mathrm{d} t}\left(\frac{\partial \varphi^{\mathrm{H}}}{\partial a_{i j}}\right)=\rho \frac{\partial^{2} \varphi^{\mathrm{H}}}{\partial a_{i j} \partial a_{m n}} \dot{a}_{m n} \\
\Rightarrow \rho \frac{\partial^{2} \varphi}{\partial a_{i j} \partial a_{m n}} \frac{\partial \Omega}{\partial\left(-X_{m n}\right)}=h \frac{\partial \Omega}{\partial\left(-X_{i j}\right)} \\
\Rightarrow \rho \frac{\partial^{2} \varphi}{\partial a_{i j} \partial a_{m n}}=\frac{1}{2}\left(\delta_{i m} \delta_{j n}+\delta_{i n} \delta_{j m}\right) h .
\end{gathered}
$$

Ainsi le paramètre d'écrouissage ne peut être fonction que des paramètres internes $a_{i j}$ ou $X_{i j}$

De la dérivation de $\Omega$ par rapport aux contraintes internes :

$$
\dot{a}_{i j}=\frac{\partial \Omega}{\partial\left(-X_{i j}\right)}=\dot{\varepsilon}_{i j}-\frac{X_{i j}^{\prime}}{\bar{X}} C(\bar{X})^{k}=\dot{\varepsilon}_{i j}-\dot{\beta}_{i j}
$$

Il résulte que le terme de restauration $r$ dans une équation type Bailey Orowan :

$$
\frac{1}{h} \frac{\mathrm{d} X_{i}}{\mathrm{~d} t}=\varepsilon-\frac{r}{h}
$$

sera lui aussi fonction uniquement des paramètres internes, puisque $\dot{\beta}_{i j}$ lié aux seules contraintes internes.

Ainsi,

$$
\varepsilon_{i j}=a_{i j}+\beta_{i j}
$$

Cette partition est importante pour analyser la réponse dissymétrique du fluage cyclique (traction compression) et permet de donner une signification physique aux $\beta_{i j}$.

Détermination de la fonction de dissipation $D$.

La fonction $D\left(\dot{\varepsilon}_{i j} \dot{a}_{i j}\right)$ a la définition suivante :

$$
\sigma_{i j}=\frac{\partial D}{\partial \dot{\varepsilon}_{i j}} \text { et }\left(-X_{i j}\right)=\frac{\partial D}{\partial \dot{a}_{i j}} .
$$

La transition de $\Omega$ à $D$ s'opère par transformation de Legendre compliquée par la forme de $\Omega$. Mais plus simplement :

$$
\begin{aligned}
& \mathrm{d} D=\frac{\partial D}{\partial \dot{\varepsilon}_{i j}} \mathrm{~d} \dot{\varepsilon}_{i j}+\frac{\partial D}{\partial \dot{a}_{i j}} \mathrm{~d} \dot{a}_{i j} \\
& \mathrm{~d} D=\sigma_{i j} \mathrm{~d} \dot{\varepsilon}_{i j}+\left(-X_{i j}\right) \mathrm{d} \dot{a}_{i j}
\end{aligned}
$$

de même : $\Omega=\Omega\left(\sigma_{i j},-X_{i j}\right):$

$$
\begin{aligned}
& \mathrm{d} \Omega=\frac{\partial \Omega}{\partial \sigma_{i j}} \mathrm{~d} \sigma_{i j}+\frac{\partial \Omega}{\partial\left(-X_{i j}\right)} \mathrm{d}\left(-X_{i j}\right) \\
& \mathrm{d} \Omega=\dot{\varepsilon}_{i j} \mathrm{~d} \sigma_{i j}+\dot{a}_{i j} \mathrm{~d}\left(-X_{i j}\right)
\end{aligned}
$$

ainsi,

$$
\mathrm{d} \Phi=\mathrm{d} \Omega+\mathrm{d} D .
$$

Ainsi, $D$ peut être choisi tel que :

$$
D=\Phi-\Omega
$$

ce qui rejoint l'analyse mathématique de Moreau [11], soit :

$$
\overline{\dot{\beta}}=\frac{X_{i j} \dot{\beta}_{i j}}{\bar{X}} \Rightarrow \overline{\dot{\beta}}=C(\bar{X})^{k}
$$

ce qui donne :

$D(\overline{\dot{\varepsilon}}, \overline{\dot{\beta}})=\left[\frac{1}{K}\right]^{\frac{1}{n}} \frac{n}{n+1}(\overline{\dot{\varepsilon}})^{\frac{n+1}{n}}+\left(\frac{1}{C}\right)^{\frac{1}{k}} \frac{k}{k+1}(\overline{\dot{\beta}})^{\frac{k+1}{k}}$

et 2 écritures de $\Phi$ :

et

$$
\Phi\left(\sigma_{i j}, X_{i j}\right)=K[\bar{q}]^{n+1}+C[\bar{X}]^{k+1}
$$

$$
\Phi\left(\dot{\varepsilon}_{i j}, \dot{a}_{i j}\right)=\left(\frac{1}{K}\right)^{n}(\dot{\varepsilon})^{\frac{n+1}{n}}+\left(\frac{1}{C}\right)^{k}\left(\frac{\dot{\dot{\beta}})^{\frac{k+1}{k}}}{2}\right.
$$

$D$ constitue une grandeur physique au même titre que la puissance dissipative $\Phi$.

Nous devons dire que dans le cas général, $\dot{a}_{i j}$ n'est pas explicite dans $D$ :

$$
\overline{\dot{\beta}}=\frac{X_{i j}}{\bar{X}}\left(\dot{\varepsilon}_{i j}-\dot{a}_{i j}\right) .
$$

Nous pouvons comparer cette analyse avec celle de Ponter [12] qui introduit $\bar{\Omega}$ dual de $\Omega$, où

$$
\bar{\Omega}=\sigma_{i j} \dot{\varepsilon}_{i j}-\Omega=\bar{\Omega}\left(\dot{\varepsilon}_{i j}, X_{i j}\right)
$$

avec

$$
\sigma_{i j}=\frac{\partial \bar{\Omega}}{\partial \dot{\varepsilon}_{i j}} \quad \text { et } \quad \dot{X}_{i j}^{\prime}=h \frac{\partial \bar{\Omega}}{\partial X_{i j}}
$$

\section{Application au fluage biaxial en chargement pro- portionnel.}

Ce travail est fait pour une histoire simple sur un échantillon recuit, le chargement $\sigma_{z z}$ et $\sigma_{z \theta}$ est fait à $t=0$.

Il permet d'écrire les rapports de proportion suivants [1] :

$$
\frac{\sigma_{z z}}{\sigma_{z \theta}}=\frac{X_{z z}(t)}{X_{z \theta}(t)}=\frac{X_{z z}^{\mathrm{st}}}{X_{z \theta}^{\mathrm{st}}}=\frac{3}{2} \frac{\dot{\varepsilon}_{z z}(t)}{\dot{\varepsilon}_{z \theta}(t)}=\frac{3}{2} \frac{\dot{\varepsilon}_{z z}^{\mathrm{st}}}{\dot{\varepsilon}_{z \theta}^{\mathrm{st}}}
$$


et ainsi, le comportement de l'A5 peut être interpréte comme l'effet d'un écrouissage cinématique avec seulement un paramètre interne $\bar{X}$ et de réduire le comportement à celui en monoaxial avec une contrainte appliquée $\bar{\sigma}$.

Ainsi $\bar{q}$ devient égal à $(\bar{\sigma}-\bar{X})$.

Les formes réduites de $\Omega, \Phi, D$ s'écrivent :

$$
\begin{gathered}
\Omega=\Omega(\bar{\sigma}, \bar{X})=\frac{K}{n+1}(\bar{\sigma}-\bar{X})^{n+1}+\frac{C}{k+1}(\bar{X})^{k+1} \\
\Phi=K(\bar{\sigma}-\bar{X})^{n+1}+C(\bar{X})^{k+1} \\
D(\overline{\dot{\varepsilon}}, \overline{\dot{a}})=\left(\frac{1}{K}\right)^{1 / n} \frac{n}{n+1}(\overline{\dot{\varepsilon}})^{\frac{n+1}{n}}+\left(\frac{1}{C}\right)^{1 / k}(\overline{\dot{\varepsilon}}-\overline{\dot{a}})^{\frac{k+1}{k}}
\end{gathered}
$$

avec :

$$
\overline{\dot{a}}=\overline{\dot{\varepsilon}}-\overline{\dot{\beta}}=\frac{X_{i j} \dot{a}_{i j}}{\bar{X}}=\frac{\overline{\dot{X}}}{h}
$$

avec enfin :

$$
\Phi=\bar{\sigma} \overline{\dot{\varepsilon}}-\overline{\bar{X}} \overline{\dot{a}} .
$$

Toutes les règles de normalité en terme de vitesse équivalente ou d'invariants sont vérifiées.

\section{Explicitation de $\rho \varphi^{\mathrm{H}}$ et de $\Phi$.}

Quand

$$
h=\frac{h_{0}}{(\bar{X})^{\beta}},
$$

le cas de notre alliage et d'autres alliages $[10,13,14]$ à haute température

$$
\overline{\dot{a}}=\frac{\overline{\dot{X}}(\bar{X})^{\beta}}{h_{0}} \Rightarrow \bar{a}=\frac{(\bar{X})^{\beta+1}}{h_{0}(\beta+1)}
$$

et ainsi, $\rho \varphi^{\mathrm{H}}$ est fonction de $\bar{a}$ et pas de $\bar{\varepsilon}$ :

$$
\rho \varphi^{\mathbf{H}}=\frac{\left[h_{0}(\beta+1)\right]^{\frac{\beta+2}{\beta+1}}}{h_{0}(\beta+2)}[\bar{a}]^{\frac{\beta+2}{\beta+1}} .
$$

Une forme proche de celle de Chaboche [15]. Elle permet de vérifier la condition suivante :

$$
\frac{\partial D}{\partial \overline{\bar{a}}}=-\rho \frac{\partial \varphi}{\partial \bar{a}}=-\bar{X}
$$

$\Phi$ prend la forme :

$$
\Phi=\overline{\sigma \bar{\varepsilon}}-\frac{(\bar{X})^{\beta+1}}{h_{0}} \overline{\dot{X}} .
$$

Pour un test de fluage biaxial, l'énergie dissipée à $t$, sera égale à :

$$
\int_{0}^{t} \Phi \mathrm{d} t=\bar{\sigma} \bar{\varepsilon}-\rho \varphi^{\mathrm{H}}=\bar{\sigma} \bar{\varepsilon}-\frac{(\bar{X})^{\beta+2}}{h_{0}(\beta+2)}=\rho T s^{(i)} .
$$
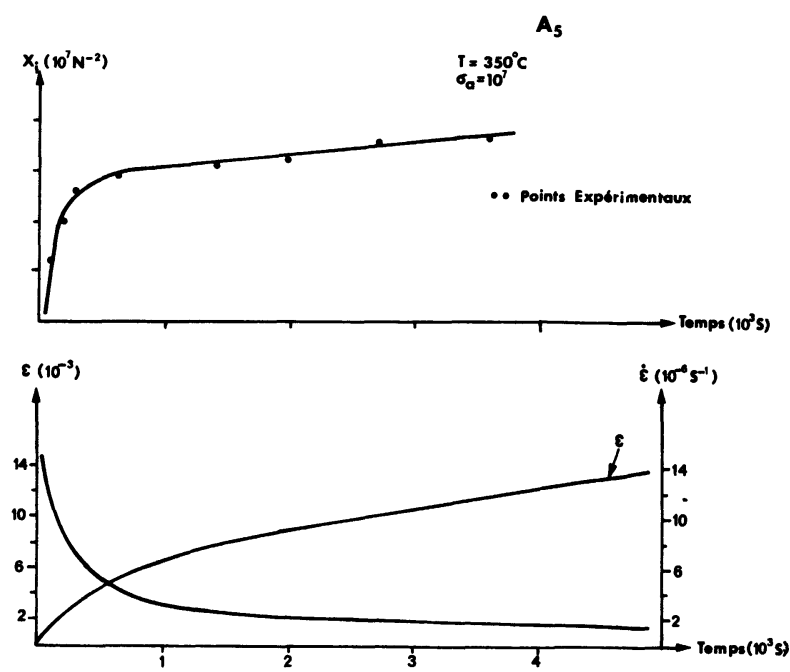

Fig. 1. - Evolution de la déformation $\varepsilon$ et de sa vitesse $\dot{\varepsilon}$ et de la contrainte interne $X_{i}$ pendant le fluage primaire au cours du temps.

[Deformation $\varepsilon$, deformation rate $\dot{\varepsilon}$ and internal stress $X_{i}$ during primary creep with time $t$.]

\section{Application à un essai monoaxial de $\mathrm{A5} \sigma_{z z}=$} $1 \times 10^{7} \mathrm{~N} / \mathrm{m}^{2} \mathrm{~T}=350{ }^{\circ} \mathrm{C}$.

Sur la figure 1, nous montrons la courbe expérimentale $\varepsilon(t)$, avec dans les mêmes conditions des mesures de contrainte interne par la "dip test technique " de Mitra-McLean [16].

En monoaxial, l'équation de la cinétique des variables internes se réduit à :

$$
\dot{a}_{i}(t)=\dot{\varepsilon}_{z z}(t)-C\left(X_{i}\right)^{k} .
$$

$C$ et $k$ ont été identifiées par le tracé de $\operatorname{Ln} \overline{\bar{\varepsilon}}^{\text {st }}$ en fonction de $\operatorname{Ln} \bar{X}^{\text {st }}$ pour différentes valeurs de $\bar{\sigma}$ en fluage biaxial :

$$
\left\{\begin{array}{l}
k=5 \\
C=3,8 \times 10^{-41} a_{i}(t) .
\end{array}\right.
$$

ce qui permet la détermination de $a_{i}(t)$.

La pente de $\operatorname{Ln} a_{i}$ en fonction de $\operatorname{Ln} X_{i}$ pendant le primaire permet de trouver $\beta=1,5$ (voir Fig. 2).

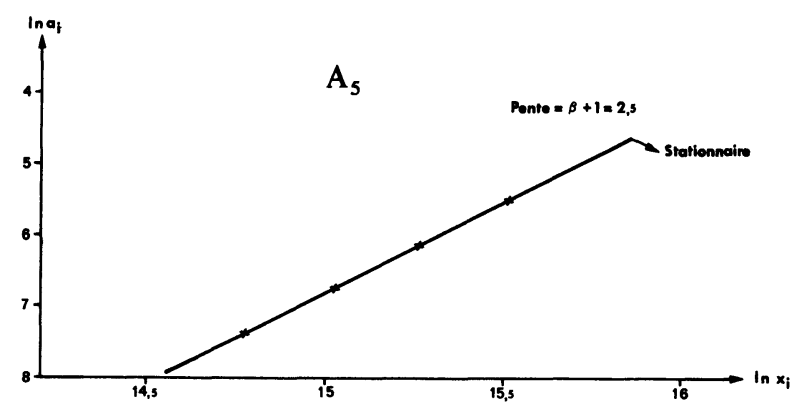

Fig. 2. - Pendant le fluage primaire, courbe de $\operatorname{Ln} a_{i}$ en fonction de $\operatorname{Ln} X_{i}$.

[Curve of $\operatorname{Ln} a_{i}$ with $\operatorname{Ln} X_{i}$ during primary creep.] 
La figure 3 montre que la puissance dissipative $\Phi$ constitue une part importante de la puissance viscoplastique $\sigma \dot{\varepsilon}$ ce qui souligne l'effet majeur des contraintes internes.

Dans la figure 4 , pendant le fluage primaire, nous montrons l'évolution du travail viscoplastique $\sigma \varepsilon$, de l'énergie libre $\rho \varphi^{\mathrm{H}}$, de $\rho T s^{(i)}$ au cours du temps. Nous devons dire que " la variable interne de déformation » est telle que :

à

$$
t=0, \quad X_{i}=0, \quad \dot{a}_{i}(0)=\dot{\varepsilon}_{z z}(0)
$$

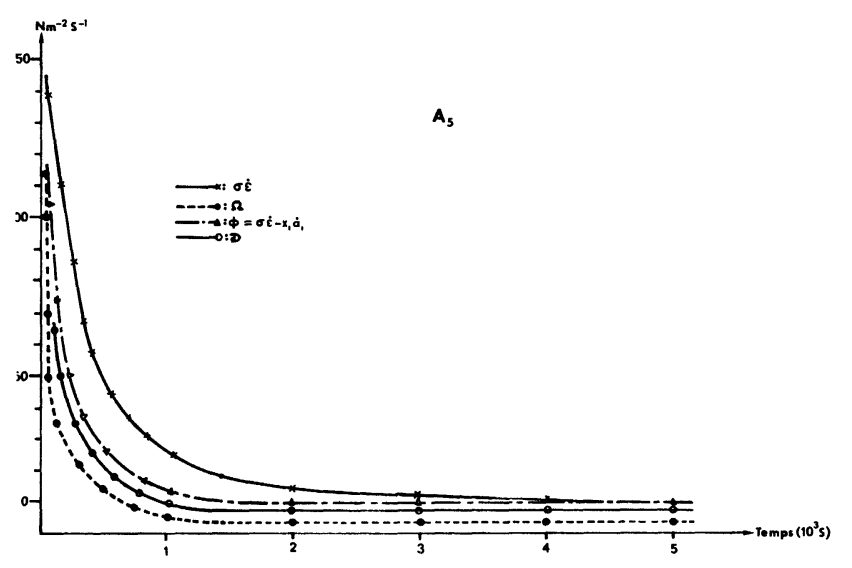

Fig. 3. - Evolution de $\sigma \dot{\varepsilon}, \Omega, \Phi$ et $D$ avec $t$.

[Evolution of $\sigma \dot{\varepsilon}, \Omega, \Phi$ and $D$ with $t$.]

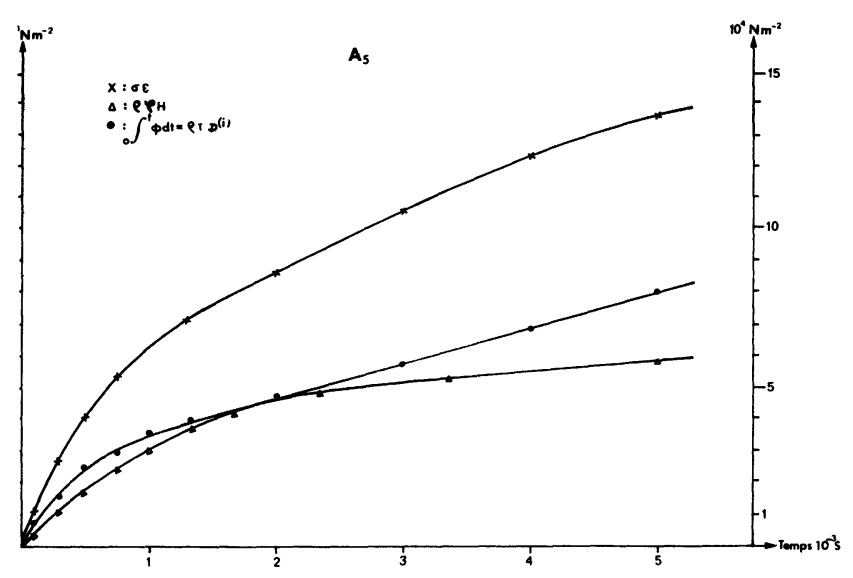

Fig. 4. - Travail viscoplastique, énergie dissipative et énergie libre $(X \rho)$ en fonction du temps.

[Viscoplastic work, dissipative energy and free energy $(X \rho)$ with $t$.]
Son évolution apparaît sur la figure 5 pour les premiers temps du fluage ( 300 premières secondes), il est difficile de séparer $\dot{\varepsilon}$ et $\dot{a}$, peut-être, la raison en est qu'il est difficile de mesurer la contrainte interne par dip test technique quand $\dot{\varepsilon}$ varie rapidement.

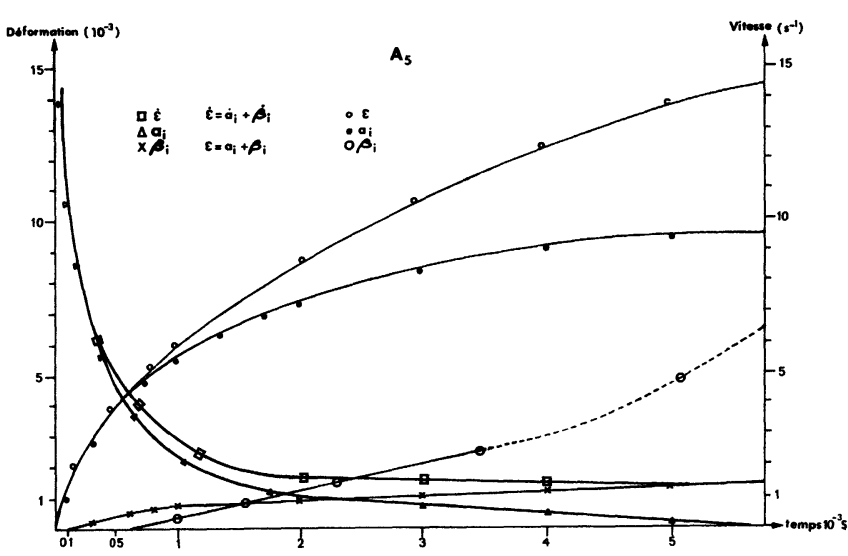

Fig. 5. - Séparation de la vitesse de déformation en 2 composantes $\dot{\beta}_{i}$ (liée aux seules contraintes internes) et $\dot{a}_{i}$, courbe de $\varepsilon, a_{i}$ et $\beta_{i}$.

[Separation of deformation rate with two components $\dot{\beta}_{i}$ : linked only with internal stresses and $\dot{a}_{i}$ : curve of $\varepsilon, a_{i}$ and $\beta_{i}$.]

\section{Conclusion.}

L'application de la théorie de Mandel permet de déterminer pour l'A5 en essais de fluage biaxiaux :

- la puissance dissipative $\Phi$,

- l'énergie libre $\rho \varphi^{\mathbf{H}}$,

- la fonction dissipative $D$.

Un travail est nécessaire au sujet de la variable interne afin de séparer les effets purs des contraintes internes de la vitesse de déformation viscoplastique. Ce modèle devra être testé pour des histoires de charges plus complexes et en particulier des chargements non proportionnels. L'extension de ces essais permettra de tendre vers la résolution de problèmes technologies réels.

\section{Remerciements.}

Je tiens vivement à remercier M. le Professeur Raniecki pour les fructueuses discussions que nous avons eues sur ce travail lors d'un séjour à l'Institute of Fundamental Technological Research de Varsovie (Pologne). 


\section{Bibliographie}

[1] Lexcellent, C., Birocheau, J. et Oytana, C., Loi de comportement en fluage biaxial de l'aluminium ( $99,5 \%$ en poids) à haute température. Revue Phys. Appl. 17 (1982) 729-736. Identification du potentiel d'écoulement stationnaire $\Omega\left(\sigma_{i j}, \alpha_{i j}^{\text {st }}, T\right)$.

[2] LAGNeBorg, R., A Modified recovery creep model and its evaluation. Met. Sci. J. 6 (1972) 127-133.

[3] MANDEL, J., Variables cachées, puissance dissipée, dissipativité normale. Colloque du Groupe Français de Rhéologie « Thermodynamique des Comportements Rhéologiques ", 7-8 décembre 1977. Sciences et Techniques de l'Armement, 53, 4e Fasc. 1979, 525-537.

[4] Birocheau, J. and Oytana, C., Role of the strain history on the flow law for high temperature creep. Mat. Sci. Eng. 56 (1982) 125-134.

[5] Coleman, B. D. et Gurtin, M. E., J. Chemical Phys. 47, no 2 (1967) 597.

[6] RANIECKI, B., Thermodynamics aspects of cyclic and monotone plasticity. Cours du Centre International des Sciences Mécaniques (UDINE-Italie). The constitutive law in thermoplasticity advanced school coordinated play, prof. Th. Lehmann. July 1982, 19-30.

[7] Ponter, A. R. S. and Leckie, F. A., Constitutive relation ships for the time dependent deformation of metals. University of Leicester, Department of Engineering Report 73-6 to appear in Trans.

[8] Rice, J. R., On the structure of stress strain relations for time dependent plastic deformation in metals. Trans. ASME, J. Appl. Mech. 37 (1970) 728-737.
[9] Orowan, E., The creep of metal. J. West Scotl. Iron Steel Inst. 54 (1946) 45-53.

[10] Robinson, D. N., Pugh, C. E. and Corum, J. M., Constitutive equations for describing high temperature inelastic behaviour of structural alloys. « Specialists » meeting on high temperature structural technology of LMFBRS, April 27-30 1976. International atomic agency IWGR/11 (October 1976) 44-57.

[11] Moreau, J. J., Sur les lois de frottement de viscosité et de viscoplasticité. C. R. Hebd. Séan. Acad. Sci. C 271 (1970) 608-611.

[12] Ponter, A. R. S., General theorems for the dynamic loading of structures for a state variable description of material behaviour. Inst. Phys. Conf. Ser. $\mathrm{n}^{\circ} 47$, Chap. I (1979) 130-141.

[13] Oikawa, H. and Sugarawa, K., Instantaneous plastic strain associated with stress increments during the steady state creep of $\mathrm{Al}$ and $\mathrm{Al}-5,5$ at Pct $\mathrm{Mg}$ alloy. Scripta Met. 12 (1978) 85-89.

[14] Goto, A., Oikawa, H. and Karashima, S., Effect of temperature and stress on work hardening and recovery rates during steady-state deformation of lead. Trans. J.I.M. 21 (1980) 15-21.

[15] Chaboche, J. L., Thèse d'état « Description thermodynamique et phénoménologique de la viscoplasticité avec endommagement ". Office National d'Etudes et de Recherches Aérospatiales. Publication $\mathrm{n}^{\circ}$ 1978-3.

[16] Mitra, S. K. and McLean, D., Work hardening and recovery in creep. Proc. Roy. Soc. (A) 295-298 (1966). 